\title{
Social Determinants of Self-Care Subsequent to Major Medical Surgery at the Central Regional Teaching Hospital in Ghana
}

\author{
William Boateng \\ Department of Sociology and Anthropology \\ College of Humanities and Legal Studies \\ University of Cape Coast \\ Cape Coast, Ghana \\ wib981@gmail.com \\ DOI//http://dx.doi.org/10.4314/gjds.v13i2.8
}

\begin{abstract}
The periods between post-discharge and full recovery can be challenging for most patients, since they have to be on self-care as they work their way back to full recovery. This study aimed to find out the experiences of discharged patients on self-care subsequent to major medical surgery at the Central Regional Teaching Hospital in Ghana. The study is informed by the qualitative research design. Experiences of twenty participants who had undergone surgery and were continuing with the recovery regimen at home were tapped into as the sample for the study. Purposive sampling technique was also adopted in selecting ten clinicians from the surgical ward to ascertain what went into preparing discharged patients to care for themselves at home after major medical surgery. Based on the findings of the study it was concluded that education, residential status, marital status, and availability and ability to convert social network into social capital constitute major social determinants of self-care. It was recommended among others that the social determinants of self-care identified above should inform plans aimed at preparing patients better to continue the recovery process on their own at home.
\end{abstract}

Keywords: Social, Determinants, Self-Care, Surgery, Discharged Patients

\section{Introduction}

Patients who undergo surgery, most often than not, have to stay in hospitals for some time to show significant evidence of recovery before being discharged to continue the journey to full recovery at home. The period between discharge and full recovery can be challenging for most patients, since they have to be on their own as they work their way back to full recovery. 
This study aimed to learn about the experiences of discharged patients continuing with treatment regimen on their own at home and the social factors affecting such self-care at home. The study is particularly important given the evidence that most patients during the post discharge period develop all sort of complications mainly emanating from snags and failure to follow rigidly medical advice received prior to discharge (Kable et al., 2004). Its importance is manifested by the fact that patients presently are discharged quicker than before because of pressure and increasing demand for hospital resources (Hull \& Erwin-Toth, 1996; LeClerc et al., 2000). Major medical surgery in this study is conceptualized as any surgery done on a patient involving a post-surgical admission at the hospital for a minimum period of two weeks.

Preparing surgical patients for the post discharge challenges can be an uphill task for clinicians for various reasons including patients perceived lack of knowledge and wrong timing of medical information (Davison et al., 2004), memory loss and unfamiliar medical terminologies (Estey et al., 1993), living in remote communities with no community-based health facilities, absence of reliable caregivers and lack of confidence to take very good care of discharged patients ( Klein-Fedyshin et al., 2005).

Patients post-surgical and discharge experiences are therefore contingent upon the package of medical preparation offered to them and/or their significant others, followup on patients' strategies, knowledge base of patients, and overall support system both from home and the communities. This study dwells on these critical areas in evaluating patients' overall journey following surgery to full recovery while on self-care at home.

The nature, timing, and form that the self-care medical education takes can go a long way in defining its success. It is evident that anxiety related to the actual admission, fatigue, and pain often makes it difficult for patients to absorb all the self-care information they are provided with while in hospital (Davison et al., 2004). Supplementing verbal teaching with written information in cases where patients and/or their significant others can read may be a big boost for positive post-discharge experience.

Unfortunately, however, health care professionals continue to provide most information to patients and their significant others verbally (Davison et al., 2004). Many health care professionals are hesitant to provide written information on self-care because of the perceived lack of knowledge of patients and their significant others especially in the developing world. Patients, therefore, stand the risk of not recalling wholly or partially the verbal medical self-care instructions received, thus exposing them to the peril of misapplying most of these self-care regimens leading to adverse recovery outcomes.

It is certain that irrespective of the form the discharge preparation takes -verbal or written - information is paramount in ensuring a successful self-care following post- 
surgical discharge. The packaging of the information should be targeted at both the patients and their significant others. A recent study has portrayed that spouses as significant others ranked their needs for information higher than patients did their needs (Davies et al., 2000). It is evident that significant others are nervous when called upon to support patients after surgical discharge because of lack of knowledge (KleinFedyshin et al., 2005). Such nervousness associated with taking care of patients after surgery at home is taken care off with the right information and preparation for selfcare. Involving significant others in the post-discharge preparation towards self-care is therefore important. This provides them with the requisite confidence to support patients more efficiently while self-caring at home.

It has been established so far that discharged patients following surgery will do well while on self-care if they are well prepared and armed with enough appropriate information. Provision of enough appropriate information to discharged patients though a necessary step, it might not necessarily be sufficient for effective self-care. The circumstance of discharged patients also has a role in determining their self-care outcomes. Unfortunately, the social determinants of effective post-surgery self-care are yet to be well established in the literature. This study therefore seeks to fill that gap by identifying the social determinants of effective self-care subsequent to major medical surgery in Ghana.

\section{The Social Determinants' Perspective on Self-Care}

Many social factors impact on how people fare at home after major surgery. Understanding these factors is critical in planning for effective policies, education, and counselling to facilitate a more meaningful and positive self-care after a major surgery. This study is informed by the "social determinants of health perspective" which is paramount in contemporary research in health care both formal and informal. Social determinants of health care are the social and economic conditions that influence health of individuals. They determine informal care and continuation of recovery regimen after surgery. Further they inform the extent to which an individual possesses the physical, social, and personal resources to informally care for their health needs (Raphael, 2004). Social determinants of health perspective thus underscore the impact of differences in access to material living conditions and resources for health (Chappell \& Penning, 2009).

Discharged patients ability to follow through effectively with medical advice obtained from health care professionals upon discharge depends on a number of variables such as the socio-economic status, education, residential status, marital status, gender, age, available and access to informal and formal community support systems. These variables intersect in diverse ways to impact self-care. This is the case because individuals' social statuses vary in terms of the quantity and quality of living such as 
physical, social, and personal resources, including income, food, housing, employment, health, and social services that are available to them (Raphael, 2006). Social determinants, therefore, have a big role in self-care after hospital discharge.

It is evident in the literature that social capital available to people impact significantly on self-care. People with better social capital are known to self-care better than others with poorer social capital. Social capital, defined as resources available to people because of their status sets, is largely influenced by one's social location. Social-economic status therefore becomes critical in this discussion. The educated and economically better off individuals residing in the big cities, for instance, are generally expected to do well when self-caring for themselves. This combined status set predisposes discharged patients to a more functional support system at home all other things being equal.

Further, marital status, gender, and age as social positions impact on self-care. The married for instance generally do better than the unmarried when self-caring because of the support from the significant other. This, however, varies depending on the gender of the married person. Married men for instance are known to receive more support from their spouses than the reverse situation because women generally do well when selfcaring than men because they are more meticulous about their health and health needs than men (Chappell \& Penning, 2009).

Ageism also becomes a social determinant of self-care because people irresponsibly give up easily on seniors and because of that denies them the needed support to recover fully at home. Even some health care professional are guilty of neglecting seniors as well. This study aims to explore how the social determinants identified above impact on self-care after medical surgery in the Ghanaian social context.

\section{Methodology}

This study was informed by the qualitative research design. Twenty participants who had undergone surgery and were continuing the recovery regimen at home were selected for the study. These discharged patients were on admission for a minimum period of two weeks following their surgeries at the Central Regional Teaching Hospital in Cape Coast, Ghana. Frequent visitations and observations made at the hospital aided in coming by the study's sample who volunteered to participate in the study. The in-depth observation and informal interactions with the target population facilitated sample elements from diverse background to determine across various social strata the social determinants of self-care after major surgery.

Participants - discharged patients - were therefore conveniently selected and those whose profile met the study's objectives purposively chosen to provide the data for the 
study. These sampling techniques were adopted because of the absence of a sampling frame covering all the potential and eligible participants for the study. In order to avoid possible biases being introduced into the selection process, however, discharged patients who fitted the selection criteria informed by gender, educational attainment, residence, and age and were willing to participate in the study were chosen. They were placed into two categories of ten participants each. The two categories were no or minimal education referring to no formal education or up to basic level education; and high education made up of secondary level education and above. Each category comprised five men and five women with varied residence and age.

Purposive sampling technique was also adopted in selecting ten clinicians from the surgical ward to ascertain what goes into preparing discharged patients to care for themselves at home after major medical surgery. Qualitative interviews were used in interacting with both categories of participants. For the participants who had undergone major surgery and recovering at home, the main issues that informed the interview process were their socio-economic status based on education, gender, marital status, residence, and availability of social capital and how they combined to affect self-care after major surgery. The clinicians were also made to react to the content of preparatory programs offered to patients, format of such programs, people involved in the preparatory programs, and frequency and length of such programs, and in-built monitoring and evaluation of such programs.

Data from the two categories of participants was analysed mainly by the use of the NVIVO. Major themes that emerged from the data were identified and related themes cross-linked to facilitate a meaningful and coherent discussion.

\section{Findings and Discussion}

Perceived lack of knowledge by patients has been identified as one of the most important factors likely to hinder the empowering of surgical patients to cope at home after surgery (Davison et. al., 2004). Perceived knowledge in this context is unrestricted to the patients but to their significant others as well since they play an important role in self-care following surgery at home. The fact that knowledge informs socio-economic status makes it an important variable to be studied as a precursor to effective selfcare after surgery. The educational level of the participants - discharged patients therefore featured prominently as a theme in their interviews. All the participants did find information received from the health care providers useful in preparing them for effective continuation of care at home after discharge. As expected, the participants with no or minimal education had to rely upon educated significant others in adhering to the regimen prescribed in support of self-care after discharge. 
The form - verbal or written - patients' preparation for self-care takes is crucial. It is explicit in the literature that health care providers in preparing patients for self-care provide information verbally (Langwade, 200o). Such method of providing information to patients might not be the best. The reasons are that with the present reduced hospitalization periods, information will not be that easy to be understood by patients (Hull \& Erwin-Toth, 1996, Hanisch, 1993). Further, the post-surgery pain, which depends on the type of surgery and the stress associated with the hospitalization, might impact negatively on how patients fare in the information session (Cupples, 1991 \& Cimprich, 1992). This evidence implies that much planning should go into self-care information sessions for better outcomes.

Unsurprisingly therefore participants overwhelmingly stressed that verbal information should be buttressed by written information as well for easy reference and also avoidance of errors following regimen handed to them. Interaction with the doctors and the nurses also pointed out that no explicit written materials are handed out to the patients upon discharge, but agreed that such an intervention is necessary albeit some cost implications. A surgical ward nurse and a doctor expressed their views on this issue:

I agree that adding written materials to the oral instructions to patients on discharge will tremendously aid the self-care process, but we are unable to do so now because it is yet to be deemed as a must do by the hospital. I suspect is more an issue of cost than any other thing, but don't quote me on this please (Surgical Ward Nurse).

It is something we have discussed and it's on the drawing board. Likely will see its implementation soon if available resources would permit that. We are aware we need to be on that road (Doctor).

Participants were thus asked about the existence of a follow-up plan to their discharge program. It became evident that such a plan was nonexistent. All the participants, however, believed that such a plan would aid positive recovery outcomes at home. The health care providers also shared the same view, but again pointed to cost as an obstacle in institutionalizing such a follow-up plan on discharged patients. A discharged patient and a surgical ward nurse expressed the following views respectively on the subject of follow ups to discharge program. These views presented are:

Follow ups would be best if situations can permit that, but I can't see it happening anytime soon. Unfortunately healthcare is not a priority in the country hence cannot see such a policy being implemented. Maybe the telephone as a means of follow ups can be tried out" - (Discharged Patient). 
"We don't have many staff to make this happen. It can happen if more staff is brought on board. The call centre system can be tried out but should be approached with caution to avoid ethical issues and privacy among others - (A Surgical Ward Nurse).

In spite of the nonexistence of a follow-up plan, patients were given specific dates to return to the hospital they had the surgery for check-ups. Patients were however not given the option to continue with the recovery process in health facilities in their communities as done in some advanced countries where list of community resources are made available to patients to help them access health facilities in their communities when necessary (Davison et. al., 2004). Such arrangement is convenient and can be cost effective as well particularly for patients with residential areas far away from the hospitals the surgeries were done.

The problem though with the above arrangement is the uneven nature of quality of health facilities in the country. Whereas urban Ghana has better health facilities, rural Ghana is worse off. This implies that advising patients on available health facilities in their communities, no doubt, will add to the overall quality of health care in the country. Implementing such a system also will mean that patients have access to their medical records to enable health care providers in the community to provide medical assistance where necessary. Unfortunately, accessing medical records in the country is difficult if not impossible. This is a highly unfortunate situation especially in an era of evidencebased medicine, which slowly but surely is making inroads into the health care system in the country.

All the participants believed that such medical leads in their respective communities would impact positively on their self-care at home. A participant on self-care in a rural community said:

Such a move will save us a lot. Since one would not have to travel more to the big towns to see doctors. It's bothersome to travel always to the big hospitals with many patients there all seeking medical attention. Such arrangement will be convenient. I hate going to the big towns but because of illness I have no choice. To have a choice will therefore be welcome - (Discharged patient).

The health care providers interviewed - doctors and nurses - however, shared the contrary view. To them community health facilities should be utilized for minor health problems, but patients should always report back to where they had the surgery when faced with complications for better health outcomes. A doctor pointed out:

Community health posts come into the picture when the cases are so basic due to lack of facilities and special expertise there. So generally I always 
recommend that post-surgery patients continuing with their recovery at home stay in touch with us to avoid further complicating health problems. Very basic issues can be followed up at health posts, I support that, but again it should be basic commensurate with the health post facilities and expertise available (Doctor).

The participants were followed-up at their respective homes after a month following discharge to learn about their experiences of self-care towards full post-surgery recovery at home. Education, which is a key variable in informing socio-economic status of individuals, received attention in ascertaining participants' self-care experiences. It was amply evident in the study that participants with high education fared better while self-caring for themselves than their counterparts with no or minimal education. This revelation did not come as a surprise because, all other things being equal, the general expectation is that with their acquired explicit knowledge would understand and administer the self-care regimen better and also appreciate why they should strictly adhere to the regimen than the participants with no or minimal education.

It was noted that whereas participants with high education understood the regimen and applied it on their own, the participants with no or minimal education had to rely on significant and in some cases generalized others in applying the regimen. Relying on others for directives adversely affected the strict adherence to the application of the regimen. This is because individuals helping out were not around always to offer assistance when needed. A participant with high education and one without shared their experiences:

"Recovering at home has been very positive due to many factors including my level of education. But following strictly my regimen has been beneficial to me. I don't need any one to push me or help me out with my treatment regimen. It all depends on me, though I appreciate all support from others" - (Educated Discharged Patient).

"I try hard to do what the doctors and nurses asked me to do, but it's difficult because I cannot read and my grandchildren who have been helping me out with my medicine are not around always. The medicines are also many making it difficult to be sorted out when they are not here. I am not sure, but I believe I would have been healed now if I was able to take the medicine on my own"(Uneducated Discharged Patient).

The above revelation is in consonance with the findings of Chappell and Penning (2009) that education as a determinant of socio-economic status features prominently in determining the outcomes of self-care after surgery. The literature has it that one's residence also influences significantly self-care outcomes (Laxminarayan et al., 2006). 
The study therefore explored the role of residential status - urban versus rural - on self-care outcomes of participants. As expected the urban residents fared well at home after surgery as compared to their counterparts in the rural areas. A discharged patient living in an urban area said: "I am pleased with my recovery process. Mainly because I am close to very good health facilities to seek answers to medical questions I have at times. Responses from these health care professionals have been helpful" - (Discharged patient with urban residence).

The rural residents on the other hand had to travel many kilometres to access quality health services in case they needed to make some health complains associated with their surgery. This revelation is obvious given the skewed nature of better health facilities in urban Ghana as compared to rural Ghana (Abdullah et al., 2011). Rural residents are thus left with little or no option than resorting to ill-equipped health centres or selfmedication (orthodox and unorthodox) in most cases to complement their treatment regimen. A discharged patient with rural residence shared his experience:

Self-care has been a mixed experience for me. I travel long distance to seek answers to my health concerns and report some complications. The high cost entailed in such travels coupled with the difficulty in having access to doctors and also the high cost of hotels or absence of places to reside if I had to stay for days in Cape Coast (urban area) have been my headache. It is an economic struggle to come and see my doctor when I have to do so - (Discharged patient with rural residence).

The study also explored how social network features in self-care after surgery. This variable was considered because the literature sufficiently has it that availability of quality social network easily translates into social capital which becomes an intermediate for quality self-care. Marital status of the participants as a source of social capital featured prominently in the study. The married individuals reported being more satisfied with their recovery trends at home than their counterparts who were not married. Even two male married participants pleaded with the research team to express on their behalf appreciation to their spouses for the support received at home on selfcare. The fact that such requests came from male participants did not come as a surprise given the prominent role rendered by women in ensuring the health needs of the family (Chappell \& Penning, 2009). There was also no difference in the satisfaction of the married individuals based on their gender. This implies that both the married men and women were satisfied with the support received from their spouses during the self-care period.

The few participants aged seventy years and above applauded the support received from their families as a form of social capital. This, however, contradicts the general notion 
that the aged receive less support during self-care based on ageism. Support from generalized others was also explored to ascertain their role in self-care. Participants were therefore made to evaluate the role generalized others such as the extended family, church, voluntary groups and the larger community rendered in their self-care process. Whereas the participants with rural residence relied more on generalized others and found them very helpful in the self-care process, the participants with urban residence rarely relied on generalized others in their self-care process. For the rural participants, the extended family and the churches were the primary generalized others who offered them the support and social capital they needed in the self-care process. The urban participants also relied more on the nuclear or immediate family members. The extended family as a social network did not emerge as significant to them.

A possible sociological justification for the above revelation is that the extended family system has fully attained an inverse relation with urbanism and urbanization in contemporary society. The inference that can be made is that the rural participants had access to a broader social network as compared to their urban counterparts. As to whether the differences in the social network translates into better self-care outcome could not be ascertained. This is because both the rural and the urban participants found their social network as valuable and would not want it changed. A wider social network therefore does not necessarily matter significantly in self-care outcomes; what matters significantly however is the individual evaluation of the social network available to them during the self-care process.

\section{Conclusion and Recommendations}

The study generally captured the following: that the educated participants understood the regimen and generally fared better with their self-care experiences than the uneducated participants who relied on others in adhering to the regimen, which impacted adversely on their self-care experiences. Information for preparing discharged patients was mainly done verbally with no specific follow-up plans in place to check on the recovery progress of the discharged patients. The patients were not given the option to continue with the recovery process in health facilities near them. Married individuals fared better on self-care than the unmarried individuals. There was, however, no genderbased difference in the satisfaction married couples derived from their spouses. The aged also fared well on self-care contrary to the notion that the aged are not well catered for by significant and generalized others. Discharged patients with urban residence were more satisfied with their self-care experiences than their counterparts with rural residence. Both, however, were satisfied with the social capital they derived from their social network. 
Based on these findings it can be concluded that socio-economic status emanating from education, urban residential status, marital status, and availability and ability to convert social network into social capital are critical social determinants for self-care following discharge after major surgery. These determinants are not ranked in an order of importance. They are all critical prerequisites in contributing to effective self-care towards full recovery of patients after surgery. These social determinants of effective self-care should occupy the centre-stage in plans aimed at preparing patients better to continue the recovery process on their own at home. The present reduction in hospital stays following surgery makes this imperative to minimize the incidence and prevalence of patients becoming sicker and thus being exposed to various risks of complications while on self-care at home.

The following recommendations are worth considering by politicians, healthcare technocrats, and the clinicians at the macro, meso and the micro healthcare levels respectively. More thorough information sessions should be organized by clinicians for discharged patients after major surgery. Easy-to-understand materials on self-care should be put together and given to patients to be followed while on self-care at home. Where possible significant others of patients should be encouraged to attend such information sessions to be prepared for the self-care support at home. This is critical in promoting the understanding of the illness experience of significant others in order to minimize stress, anxiety and encourage empathy thus facilitating meaningful involvement to help patients out at home. Individuals recovering at home may not be in a position to effectively take care of their needs hence relying on others. Involving significant others in preparing patients for self-care should therefore be prioritized irrespective of the patient's educational level and social circumstance.

The way and manner the information is passed on to patients is also critical. Patients and their significant others are more likely to benefit from information from the health care providers if the information is well packaged to suit varied levels of knowledge. Surely a one size fits all approach will fail hence the need to vary information delivery to suit peculiarities of patients. This is imperative because where patients and significant others are unfamiliar with medical terminologies, successful self-care after surgery has been knotty (Estey, 1993). The packaging of information is therefore as important as the information itself.

There is the need for the introduction of a follow-up plan for discharged patients. This, undoubtedly, will go a long way in ensuring effective self-care and quality recovery outcome. Follow-up can take varied forms. Physical visitation is one, but not easily feasible because of its cost implications. Community health nurses working in the communities, however, can be brought on board to do such follow-ups. Their involvement, however, is unlikely because of their overloaded responsibilities in the 
communities as primary health care providers. The telephone can also be utilised for follow-up purposes.

Healthcare call centre system can be introduced in the country. This system is worth a try because most Ghanaians at the moment have access to mobile phones, which predetermines a functional healthcare call centre system. The call system will offer patients on self-care the opportunity to phone and make inquiries about their health needs and seek answers to their health questions. If such a system is introduced and made to run effectively it would ease the pressure on the heavy traffic of people to hospitals on daily basis. Such call centres should be manned by professionals and best practices from countries around the world taken into account to ensure a successful system. In an era of mobile-technology it is very critical that full use is made of it to improve the living standard of the people. In Ghana, mobile phone is now a status symbol, hence the possibility of such healthcare system being very successful in the country.

Quality healthcare facilities should be extended to all areas in the country. It is no coincidence that rural residence on self-care fared poorer with their self-care experiences as compared to the experiences of their counterparts with urban residence as shown in this study. Though it is a reality that rural healthcare system cannot be placed on the same pedestal as the urban healthcare system, more efforts need to be made to bridge the "healthcare quality gap" somehow. It should be feasible for every district in the country to boost of at least a good healthcare facility to take care of the health needs of the people in the district. Already some districts have very good healthcare system, others do not have, hence the need to make it a policy to ensure that all districts have some requisite standard of healthcare system at their disposal. Such a move holds the potential to ease the pressure currently being borne by the urban healthcare system, which is also rendering the overall healthcare system ineffective.

It is worthwhile looking into the recommendations because there are presently no special programs in place within the Ghana Health Services for strengthening or improving upon present surgical services at the district hospitals (Abdullah et al., 2011). This is unfortunate because improving surgical and obstetric capacity in district hospitals in developing countries is deemed as cost-effective from public health standpoint in the long run (Laxminarayan et al., 2006). 


\section{References}

\section{Abdullah, F., Choo, S., Hesse, A., Abantanga, F., Sory, E., Osen, H., Ng, J., McCord, C.,Cherian, M., Fleischer-Djoleto, C., and Perry, H. (2011).} Assessment of surgical and obstetrical care at 10 district hospitals in Ghana using on-site interviews. Journal of Surgical Research. Vol. 171 (2), pp. 461-466.

Chappell, N., and Penning, M. (2009). Understanding health, healthcare, and health policy in Canada: Sociological perspectives. Toronto: Oxford University Press.

Cimprich, B. (1992). A theoretical perspective on attention and patient education. Advances in Nursing Science, 14, pp. 39-51.

Cupples, S. A. (1991). Effects of timing and reinforcement of preoperative education on knowledge and recovery of patients having coronary artery bypass graft surgery. Heart and Lung, 20, pp. 654-66o.

Davies, N. (2000). Carers' opinions and emotional responses following cardiac surgery: Cardiac rehabilitation implications for critical care nurses. Intensive Critical Care Nursing, 16 (2), pp. 66-75.

Davison, J., Moore, K., MacMillan, H., Bisaillon, A., Wiens, K. (2004). Patient evaluation of a discharge program following a radical prostatectomy. Urologic Nursing, 24(8), pp. 483-489.

Estey, A., Kemp, M., Allison, S., and Lamb, C. (1993). Evaluation of a patient information booklet. Journal of Nursing StaffDevelopment, 9, pp. 278-282.

Hanisch, P. (1993). Information needs and preferred learning time to receive information for phase II cardiac rehabilitation patients: What CE instructors need to know. The Journal of Continuing Education in Nursing, 24. pp. 82-89.

Hull, T. L., and Erwin-Toth, P. (1996). The pelvic pouch procedure and continent ostomies: Overview and controversies. Journal of Wound, Ostomy and Continence, Nursing. 23. pp. 156-165.

Kable, A., Gibberd, and Spigelman, A. (2004). Complications after discharge for surgical patients. ANZ Journal of Surgery, 74 (3), pp. 92 - 97.

Klein-Fedyshin, M., Burda, M., Epstein, B., and Lawrence, B. (2005). Collaborating to enhance patient education and recovery. Journal of the Medical Library Association, 93(4), pp. 440-445. 
Langwade, J. (2000). The experience of early discharge after TURP. Professional Nurse, 15,.pp. $758-760$.

Laxminarayan, R., Mills, A., Breman, J., Measham, A., Alleyne, G., Claeson, M., Jha, P., Musgrave, P., Chow, J., and Shahid-Saller, S. (2006). Advancement of global health: key messages from the Disease Control Priorities Project. The LANCET. Vol. 367 (9517), pp. 8-14.

LeClerc, C., Wells, D., Craig, D. and Wilson, J. (2002). Falling short of the mark: Tales of life after hospital discharge. Clinical Nursing Research, 11 (3), pp. 242-63.

Raphael, D. (2004). Social determinants of health: Canadian perspectives. Toronto: Canadian Scholars' Press.

Raphael, D. (2006). Social determinants of health: An overview of concepts and issues, In Raphael, D., Bryant, T., and Rioux, M., (eds), Staying Alive: Critical Perspectives on Health, Illness, and Health Care. Toronto: Canadian Scholars Press, pp. 115-38. 